\title{
Posibles riesgos de corrupción. Un desafío ético para el Ejército Nacional ${ }^{1}$
}

https://doi.org/10.21830/9789585377127.05

\author{
Andrés Hernando Matiz Rojas ${ }^{2}$ \\ Luisa Fernanda Morales Osorio ${ }^{3}$ \\ Escuela Militar de Cadetes "General José María Córdova”
}

\section{Resumen}

Este capítulo de libro inicialmente trata acerca de los aspectos éticos y psicosociales de la corrupción. Seguido de ello se hace mención de la relación entre este fenómeno psicosocial y el sector público y privado. Finalmente, se determina el riesgo de corrupción en la Fuerza Pública. Para desarrollar este trabajo se empleó el método cualitativo de carácter inductivo, en el entendido de que este método es el adecuado para estudiar las relaciones, las percepciones y las representaciones, con un alcance además descriptivo que busca especificar las propiedades, las características y los perfiles de personas, grupos, comunidades, procesos, objetos o cualquier otro fenómeno que se someta a un análisis. Los resultados muestran que debido a la magnitud, capacidad operativa, cantidad de personas y presupuesto que tiene el Ejército Nacional, se pueden presentar diferentes riesgos de corrupción, razón por la cual la institución cuenta con un sistema robusto de

1 Este capítulo presenta los resultados colaborativos de dos proyectos de investigación: (1) "Ética militar en entornos complejos de seguridad y defensa: lecturas y aportes desde la experiencia de las Fuerzas Militares de Colombia", del grupo de investigación Masa Crítica, de la Escuela Superior de Guerra "General Rafael Reyes Prieto", Colombia, categorizado en B por Minciencias y con código de registro COL0123247, y (2) "Ética y corrupción", del grupo de investigación en Ciencias Militares, de la Escuela Militar de Cadetes "General José María Córdova”, Colombia, categorizado en B por Minciencias y con código de registro COL0082556. Los puntos de vista pertenecen a los autores y no reflejan necesariamente los de las instituciones participantes.

$2 \mathrm{PhD}$ (c) en Estudios Internacionales de Paz, Conflictos y Desarrollo de la Universitat Jaume I. Ingeniero de la Universidad de Ciencias Aplicadas y Ambientales. Investigador de la Facultad de Ciencias Militares de la Escuela Militar de Cadetes "General José María Córdova”, Colombia. Orcid: https:/orcid. org/0000-0001-6801-9064 - Contacto: andres.matiz@esmic.edu.co

3 Magíster en Seguridad y Defensa Nacionales de la Escuela Superior de Guerra "General Rafael Reyes Prieto”. Profesional en Relaciones Internacionales y Estudios Políticos de la Universidad Militar Nueva Granada. Asesora de investigación formal y formativa de la Escuela Militar de Cadetes “General José María Córdova”, Colombia. OrCid: https://orcid.org/0000-0002-6114-8354 - Contacto: luisa.morales@esmic.edu.co 
administración de riesgos internos. Se concluye que es importante que los uniformados del Ejército Nacional no solo memoricen las enseñanzas de ética militar, sino que también las apropien con diferentes metodologías innovadoras.

Palabras clave: conducta; corrupción; Ejército Nacional de Colombia; ética; riesgo; transparencia.

\section{Introducción}

El Ejército Nacional de Colombia es una de las instituciones más grandes del país y también una de las que más confianza genera. Dado que está distribuida por todo el territorio nacional, llega a los lugares más lejanos del país, pero este hecho también la convierte en una institución con riesgo de corrupción, debido a la gran cantidad de presupuesto, personal y procesos que maneja.

Esto también dificulta en parte el trabajo de seguimiento y control que pueda hacer la entidad a cada una de sus unidades o a cada una de las personas que la integran. Por esta razón, al final, la ética militar es la que prevalece en el momento de no cometer un hecho de corrupción. Precisamente, en el Manual fundamental del Ejército MFE 1.0, el Ejército Nacional (2017) explica

la importancia de la aplicación ética del poder militar, la importancia de la ética institucional, esta vista como uno de los principios institucionales los cuales están al mismo nivel del respeto de la Constitución y la ley y la fe en la causa, donde la ética debe estar en todas las actuaciones y comportamiento militares, la cual también aporta a los valores del Ejército donde se refieren a la transparencia como honor y rectitud en las acciones que se emprenden por la patria, siendo impenetrables ante la corrupción y actuando según la Constitución Política de Colombia y la ley, bajo el lema: "La ética y la integridad me hacen transparente", por otra parte también el Código de Honor del Soldado Colombiano la resalta y explica que la "ética es mi única regla para tomar decisiones”. (pp. 42-23)

Esto evidencia que la ética es tan importante para la institución como el respeto a la Carta Magna de la República de Colombia y, por lo tanto, para el ejercicio de la carrera y el actuar militar, que están enmarcados en los principios, valores y códigos de honor de la Fuerza.

De esta manera, el Ejército Nacional de Colombia (2017) explica la ética militar como un reto que surge debido al ambiente operacional y que recae 
sobre los hombros de los líderes de las pequeñas unidades. Estos líderes son los responsables de mantener la disciplina y asegurar que la conducta de los soldados permanezca dentro de los límites éticos mientras defienden la patria y aceptan también ser los guardianes de la ética. La ética es la única regla, la regla de oro, para tomar decisiones (Ejército Nacional de Colombia, 2017, pp. 44-43).

Esta perspectiva define la ética como un componente intrínseco al deber ser de un soldado y lo expone en un ambiente operacional. Sin embargo, si bien este es el mayor ambiente donde los integrantes de la Fuerza van a realizar sus actividades, existen otros tipos de ambientes en los cuales también se deben tener en cuenta los aspectos éticos, como los ambientes administrativos de la Fuerza, donde hay mayor riesgo de corrupción debido a los procesos que se manejan.

Según Transparencia por Colombia (2019a), la corrupción en Colombia es un fenómeno que afecta la legitimidad y la confianza en el Estado. Rompe y corrompe el comportamiento racional del Estado. De acuerdo con la definición de Transparencia Internacional, la corrupción es el abuso de poder o de confianza por parte de un actor para obtener beneficios particulares en detrimento de los intereses colectivos (parr. 1).

Como consecuencia, según Presidencia (2019), la lucha contra la corrupción es y seguirá siendo una prioridad del Gobierno Nacional y así lo manifestó el presidente de la República, Iván Duque Márquez, durante los primeros cuatro meses de gobierno, en los cuales primó la equidad como un objetivo de trabajo (parr. 1). De acuerdo con el presidente, "corrupción no es solamente el que peca por la paga o el que paga por pecar; la corrupción es la degradación moral del individuo, la degradación moral de la sociedad, y por eso la sociedad no puede tener relativismo moral frente a ninguna forma de delito" (parr. 4).

Es importante tener en cuenta la reflexión que hace el presidente, donde relaciona la corrupción con una degradación moral del individuo y de la sociedad. En este sentido, es importante tener en cuenta que además de una política fuerte de administración de riesgos, también se debe fortalecer la ética en los integrantes del Ejército Nacional para que este tipo de degradación no 
ocurra. Es por esto que él manifiesta que la corrupción ha planteado graves problemas y amenazas para la estabilidad y la seguridad de las sociedades al socavar las instituciones y los valores de la democracia, la ética y la justicia. Por ello es imperativo asegurar el futuro y la paz en el país, consolidando la ética como eje fundamental en la vida del militar y promoviendo el ejemplo de ciudadano que debe ser (Mindefensa, 2016).

El párrafo anterior, además de establecer la ética como un eje fundamental e intrínseco de la vida militar, también exige al uniformado ser un ejemplo para la sociedad, una persona que no se degrade ni tampoco degrade a las Fuerzas Militares. Consciente de esto, el Comando General de las Fuerzas Militares, mediante la Disposición 036 de 2016, aprobó la creación del Manual de generalidades éticas para la vocación militar, el cual se debe implementar en todos los niveles de mando y en las escuelas de formación y capacitación de las Fuerzas Armadas.

Aunado a esto, el Ejército Nacional presentó el Código de ética institucional del Ejército 2020, el cual busca dar cumplimiento a la misión constitucional. De esta manera, se actualiza el Código de Ética Institucional para complementar y fortalecer las normas de conducta de los soldados miembros del Ejército, los valores, principios y reglas mínimas de comportamiento que se deben aplicar en el actuar individual e institucional, para que aumente la preservación de la legitimidad institucional. Además, este Código de ética es una herramienta para fortalecer en la institución la cultura de la integridad, la prevención de las malas prácticas y el fomento de la transparencia (Ejército Nacional de Colombia, 2020a, pp. 5-7).

Así, se puede pensar que una de las apuestas que tiene el Ejército Nacional consiste no solo en fortalecer los procesos de administración de riesgo, sino también fortalecer la ética de los uniformados de la institución para que estos no incurran en actos de corrupción. Teniendo en cuenta lo anterior, surge la siguiente pregunta de investigación: ¿Cómo la ética puede aportar a disminuir el riesgo de corrupción en el Ejército Nacional de Colombia? Para dar respuesta a esta pregunta, se estableció como objetivo de la investigación establecer la forma en que la ética aporta a disminuir el riesgo de corrupción en el Ejército Nacional. 


\section{Marco teórico}

En la actualidad, ningún Estado, región o bloque de cooperación es ajeno a los constantes cambios del sistema internacional y de la realidad que a todos involucra: la corrupción. Esta es una realidad que permea a la gran mayoría de las naciones y que se materializa por las prácticas corruptivas que realizan las distintas instituciones públicas y privadas que las conforman y que son aparentemente difíciles de controlar, pese a los esfuerzos combinados que se hacen también por algunos actores sociales y estamentos del Estado. Si bien es cierto que la corrupción es un fenómeno complejo que permite distintas apreciaciones, nos centraremos en el presupuesto de defensa y la sociedad del sector defensa (Estévez, 2005).

En términos generales, la corrupción se ha convertido en el escenario ideal para fomentar la inestabilidad institucional y para que aumente el desgaste de las relaciones entre las instituciones del Estado y, en esa misma instancia, todos los actores de la sociedad. Lo anterior conlleva una pérdida de legitimidad que varios gobiernos han experimentado, así como la ineficiencia de la burocracia y con ello la polarización del poder, las cuales constituyen algunos de los problemas que trae consigo la corrupción. Además, el creciente desarrollo y conformación de bloques de cooperación pone como uno de los temas principales en la agenda de seguridad del Estado la lucha contra la corrupción, no solo en términos nacionales, sino también transnacionales.

A los problemas mencionados se suma el avance y la mejora continua de las tecnologías de la información y las redes de comunicación, que aumentan los efectos de los ejercicios corruptos. Desde el punto de vista de la economía, la creación de una red electrónica internacional a través de la cual opera el nuevo sistema financiero ha presentado un sinnúmero de interrogantes acerca de cómo regular, controlar y evitar posibles fraudes informáticos. En este sentido, la experiencia de otros Estados muestra que la corrupción se vale de los paraísos fiscales, la existencia de monopolios, la evasión impositiva, la licitación de grandes obras civiles, la privatización de empresas estatales, etc., para nutrirse de los recursos nacionales e internacionales públicos y privados (Estévez, 2005). 
Ahora bien, desde el punto de vista social, la corrupción ha sido estrechamente relacionada con escenarios de extrema pobreza, los cuales son utilizados para sacar provecho y/o garantizar la adjudicación de presupuesto para inversión social o mejora de la infraestructura sin que se materialice o se ejecute. Asimismo, los conflictos entre las diversas jerarquías sociales y su involucramiento en luchas por poder y beneficios personales han demostrado la necesidad de repensar la estructura, la relación y la distancia de los distintos estratos sociales. En orden a tener una mejor conceptualización de esta faceta de la corrupción, la literatura le otorga un papel preponderante al intercambio de la información y al fortalecimiento de la confianza interpersonal como medios para fortalecer el vínculo social entre los distintos actores de la sociedad (Estévez, 2005).

De acuerdo con Sandoval (2016), la mayor parte de la literatura académica sobre corrupción se fundamenta en dos hipótesis metodológicas erróneas. La primera es que se cree que el origen de este fenómeno se da en el gobierno y en las instituciones que conforman el sector público del Estado. En consecuencia, todo lo que tiene que ver con el Estado y con el gobierno es estrechamente relacionado con la corrupción y la ineficiencia en los órganos de control. La segunda hipótesis, que tiene el mismo grado de gravedad, es que se tiene la percepción de que la corrupción es un comportamiento de cada persona.

En este sentido, la Organización de las Naciones Unidas (ONU), en su Programa Global contra la Corrupción, afirma que la corrupción es un "fenómeno social, político y económico complejo que afecta a todos los países. La corrupción socava las instituciones democráticas, frena el desarrollo económico y contribuye a la inestabilidad gubernamental" (Oficina de Naciones Unidas contra la Droga y el Delito [UnOdC], 2020).

Este concepto confluye con el de Transparencia por Colombia (2019b), que lo describe como el "abuso de posiciones de poder o de confianza, para el beneficio particular en detrimento del interés colectivo, realizado a través de ofrecer o solicitar, entregar o recibir bienes o dinero en especie, en servicios o beneficios, a cambio de acciones, decisiones u omisiones" (Transparencia por Colombia, 2019b), y con el concepto de Johnston (2006), que lo define como "el abuso del poder público para la ganancia privada que amenaza el interés público” (p. 12). 
En todas estas formulaciones predomina un enfoque vinculado con las "teorías de la modernización", las cuales consideran que la corrupción es resultado del "subdesarrollo", del dirigismo económico o de la falta de una llamada "cultura de la legalidad". Tales planteamientos han logrado generar un amplio consenso tecnocrático que, como remedio para todo tipo de corrupción, propone modernizar burocracias, mejorar incentivos de mercado y educar a la sociedad para así ascender rápidamente hacia los añorados primeros lugares del Índice de Percepción de la Corrupción de Transparencia Internacional (Sandoval, 2016).

La interpretación actual de la corrupción tiende a ser confundida entre lo público y lo privado. Esto hace que sea necesario examinar más detalladamente este concepto. La corrupción implica al hombre desde dos ópticas distintas: una se puede llamar ex intra ('desde dentro') y otra ex extra ('desde fuera'). Sin embargo, la definición moderna implica ciertos problemas de extensión. ¿Dónde están los límites claros entre el rol público y el privado; Estado y sociedad; gobierno y Estado; política y administración; intereses, derechos y obligaciones individuales y colectivas? (Estévez, 2005).

\section{Aspectos éticos y psicosociales de la corrupción}

Algunos estudios que se han realizado acerca de la aproximación psicológica de las personas sobre conductas deshonestas y poco éticas han arrojado que es una situación que tiende a aumentar. En este sentido, también se ha demostrado que estos comportamientos y estos actos tienen una tendencia de aceptación en la sociedad dado que es un fenómeno que ha tenido una erosión gradual de algunos criterios éticos (Gino \& Bazeman, 2009).

Lo anterior permitiría comprender la institucionalización de la corrupción: si un individuo percibe que sus conductas (catalogadas como corruptas) son normales en su grupo, entonces ellas no constituirán una violación de las normas del grupo, y es entonces cuando se presentará un reforzamiento de la conducta y por consiguiente aumentará la brecha para tratar de reducirlas posteriormente (Julián \& Bonavia, 2017).

Al respecto, Mishra (2006), en su artículo "Persistencia de la corrupción: algunas perspectivas teóricas" resalta que "es la omnipresencia de las conductas 
corruptas la que contribuye a su persistencia. Es decir, en aquellas sociedades donde comportarse de manera corrupta se convierte en algo normalizado y conveniente, las políticas e incentivos contra la corrupción pierden toda su eficacia" (Mishra, 2006).

Consecuente con lo anterior, estas conductas deshonestas pueden influir de tres formas distintas la ética de quienes están observando:

1. Al estar presenciando aquellas prácticas y al percibir que no existe algún tipo de "castigo", siente o cree que las posibilidades de que sea puesto en evidencia disminuyen.

2. Si evidencia que estas prácticas son llamativas, quien está observándolas o presenciándolas tiende a evaluar su propio juicio ético, lo que conllevará que reevalúe la ejecución de este tipo de conductas en un futuro.

3. Presenciar un acto deshonesto lo puede llevar a replantear las normas sociales establecidas respecto a la deshonestidad.

Esto último está relacionado con lo que propone la "teoría centrada en la norma" (Cialdini et al., 1990), que explica que el contexto social determina cuál de las normas (descriptivas y prescriptivas) atiende un individuo en un momento particular y cómo esa norma afecta el comportamiento inmediato. Las normas descriptivas serían aquellas que especifican qué es lo que hace la mayoría de la gente en una situación concreta, mientras que las normas prescriptivas aclaran qué comportamientos son aprobados o desaprobados por las personas (Julián \& Bonavia, 2017).

Varios de los estudios que se han realizado en el campo de la ética comportamental arrojan que las personas son "demasiado buenas" como para entender y diferenciar una conducta por ética si se está recibiendo algún beneficio. Lo anterior se explica en el sentido de que la corrupción, además de ser un acto de carácter individual, también es de carácter social; en este sentido, en un entorno de competencia continua, una persona gana a costa de otra. El posible conflicto de intereses que pueda surgir en el grupo vencedor se ve mermado por el hecho de que las personas consideran que sus decisiones son necesarias y 
están fundamentadas en poderosos motivos, incluso a sabiendas de que perjudican a otras personas (Díaz, 2003).

Consecuente con lo anterior, algunos investigadores optan también por explicar la corrupción desde el punto de vista de las deficiencias en los valores éticos de la sociedad. Por un lado, la corrupción representaría la dificultad en el desarrollo ético y moral que supone un obstáculo para que los individuos tengan la capacidad de reconocer un acto o una conducta inadecuada o deshonesta. Y por otro lado, se presentan algunas disparidades respecto a la lucha contra la corrupción, dado que se perciben enfoques diferenciales y/o parciales, así como la omisión de políticas de prevención que promuevan la integridad en el sistema público (Diego, 2018).

\section{La corrupción, el sector público y privado}

Una deliberación exhaustiva respecto a la manera de impedir la corrupción y evitar que los individuos que ocupan cargos públicos practiquen actitudes indebidas conduce a la afirmación de que la causa inevitable de la corrupción es la conducta deshonesta del actor público, y esto sucede porque el individuo decide realizar la acción corrupta. Si el individuo ya ha decidido cometer un acto corrupto, planeará la manera de evadir las normas y los mecanismos de control. En todo caso, está en él la decisión de actuar o frenarse. Y esto último puede lograrse gracias a la sensibilización, al desarrollo de la conciencia, a la madurez de juicio, resultado del establecimiento de unos principios internos y un dominio del carácter. Son los principios y el carácter los que impiden o hacen actuar a una persona, y la ética es la disciplina que los muestra. Así llegamos al terreno de la ética, la cual, al ser aplicada al ámbito público, pasa a denominarse "ética pública” o "ética para la administración pública” (Bautista, 2005).

La ética, al referirse al ámbito público, implica necesariamente relacionarse con la política, es decir, no se limita a los funcionarios públicos. Un buen gobierno no solo requiere funcionarios responsables, sino también políticos responsables, puesto que son estos últimos principalmente quienes gozan del máximo margen de autonomía en las decisiones y de estas decisiones depende a su vez la actuación de los principios (Bautista, 2005). 
La ética pública es un elemento importante para hacer contrapeso no solo a la corrupción, sino también a las distintas actitudes antiéticas, pues inyecta un conjunto de principios y valores que revitalizan, por un lado, a las instituciones públicas y, por otro, a los servidores públicos, entendiendo como tales a aquellas personas que ocupan un cargo público y sirven al Estado: políticos y funcionarios (Bautista, 2005).

Más que un problema de falta de competencia, afirma Sandoval (2016), la corrupción estaría ligada a un vacío en la regulación que permitiría constantes abusos de poder. Este enfoque estructural de la corrupción se sustentaría en tres factores fundamentales: (1) una dominación social que se basa en diferencias de poder, (2) la existencia de impunidad en las altas esferas del poder y (3) la exclusión de los ciudadanos de los mecanismos de participación democráticos. Además, la corrupción ya no solo se da en el ámbito estatal - hipótesis tradicional de estudio entre los investigadores-, sino también en el ámbito privado. En términos similares, Vicuña et al. (2006) encontraron que un tercio de una muestra universitaria atribuía los actos corruptos al abuso de poder de la burocracia.

En un estudio llevado a cabo en la ciudad de Buenos Aires, Boniolo (2010) comprobó que la corrupción era percibida como algo común por los entrevistados y que no solo ocurría en las altas esferas de poder, sino también en instituciones privadas. Las prácticas corruptas se ven favorecidas por la ausencia de controles efectivos sobre la élite económico-política y actúan siguiendo sus propias reglas (Boniolo, 2010). De esta manera, la corrupción formaría parte de las instituciones y se reproduciría a lo largo del tiempo, menoscabando la estructura social y mermando la percepción favorable de los ciudadanos sobre sus instituciones estatales.

\section{Riesgo de corrupción en la Fuerza Pública}

Es importante comenzar aclarando que en la gestión pública existen diferentes tipos de riesgo, los cuales son diferentes, pero en algunos casos se confunden en una misma categoría: los riesgos de gestión y los riesgos de corrupción. Específicamente, el Departamento Administrativo de la Función Pública (2018) los conceptualiza de la siguiente forma: 
1. Riesgos de gestión: posibilidad de que suceda algún evento que tendrá un impacto sobre el cumplimiento de los objetivos. Se expresa en términos de probabilidad y consecuencias.

2. Riesgo de corrupción: posibilidad de que, por acción u omisión, se use el poder para desviar la gestión de lo público hacia un beneficio privado. (p. 8)

Teniendo en cuenta lo anterior y el contexto estratégico de la administración de riesgos del Departamento de Planeación del Ejército Nacional (2020b), se deben analizar los diferentes factores internos y externos en el momento de hablar de la administración de riesgos para prevenir o contrarrestar algunas de las 204 causas (45 externas y 159 internas) que ponen en riesgo el contexto estratégico de la institución (figura 1).

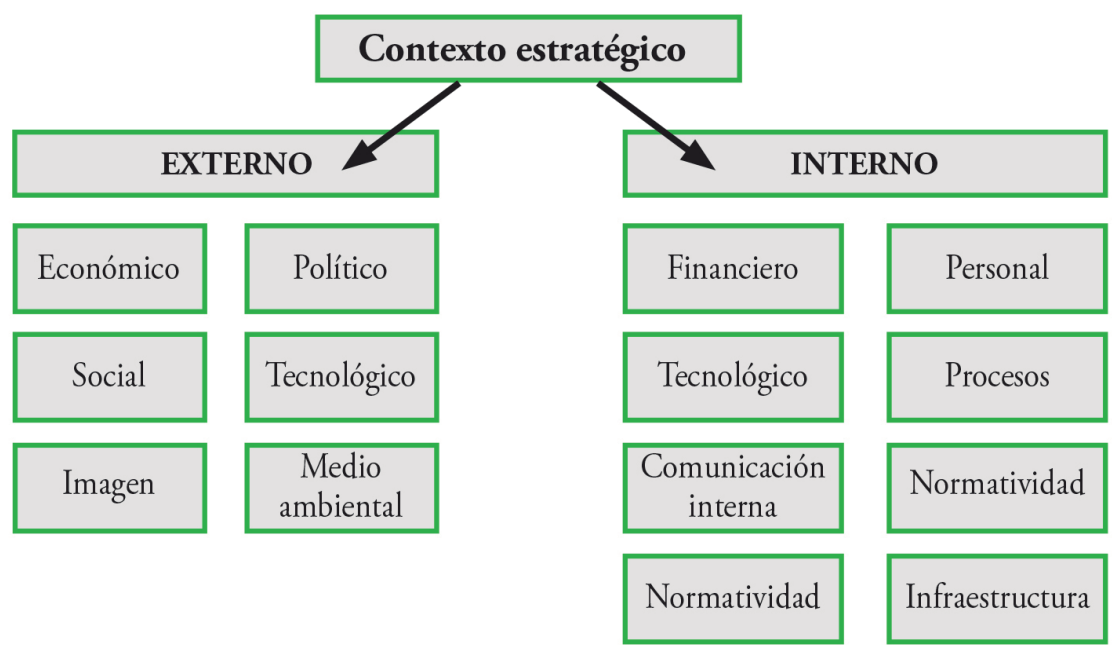

Figura 1. Contexto estratégico de la administración de riesgos.

Fuente: elaboración propia con base en la Matriz Estratégica de Riesgos del Departamento de Planeación del Ejército Nacional de Colombia (2020b).

Si bien los factores externos son importantes, la institución no puede controlar la mayoría de las causas derivadas de estos, ya que son variables que nacen o se crean dependiendo del contexto del país y el Ejército responde ajustándose a este contexto. Sin embargo, los factores internos sí pueden ser 
predecibles y debido a esto se pueden realizar estrategias de prevención con el fin de mitigar la ocurrencia de las causas de riesgo interno, ya sean riesgos de gestión o de corrupción (figuras 2 y 3 ).

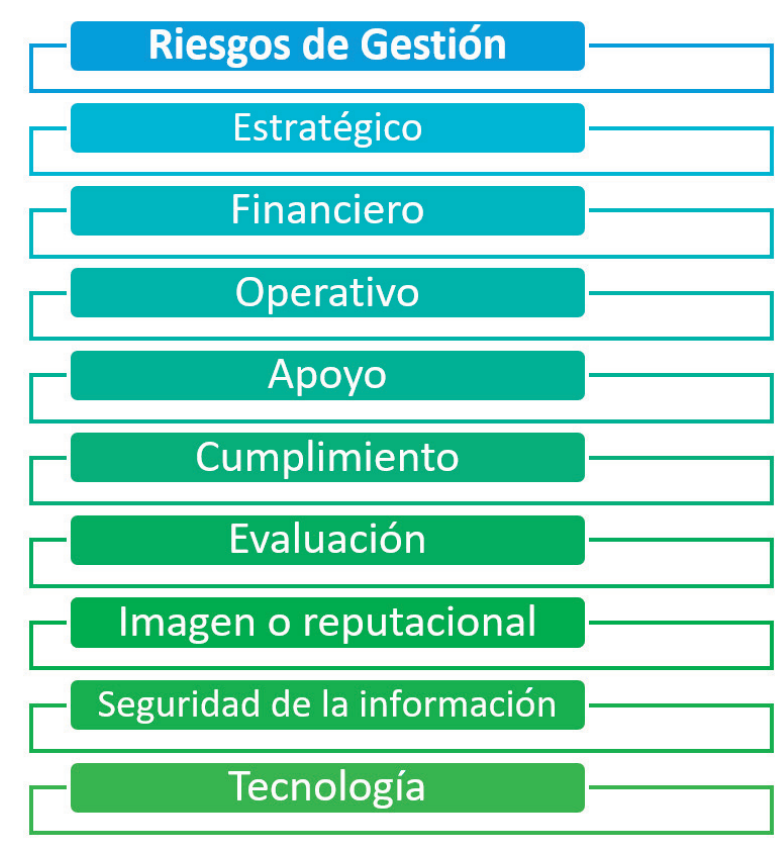

Figura 2. Riesgos de gestión.

Fuente: elaboración propia con base en Informe anual de riesgos vigencia 2019 (Departamento de Planeación del Ejército Nacional de Colombia, 2020a).

Las figuras 2 y 3 del Departamento de Planeación del Ejército (2020a) exponen el contexto estratégico de los procesos del Sistema Integrado de Gestión y los riesgos de gestión y corrupción. Específicamente, se identificó el mayor nivel de riesgo en el tipo operacional, con el $41 \%$, seguido del riesgo de corrupción, con el 26 \% (pp. 8-3). Esta cifra evidencia un nivel de riesgo alto comparado con los demás riesgos de gestión y muestra los procesos susceptibles de corrupción en la institución, lo cual llevó al Departamento de Planeación del Ejército (2020a) a establecer que el impacto que se debe colocar en la evaluación es de riesgo residual, teniendo en cuenta la calificación del riesgo de corrupción impactado (p. 6). 


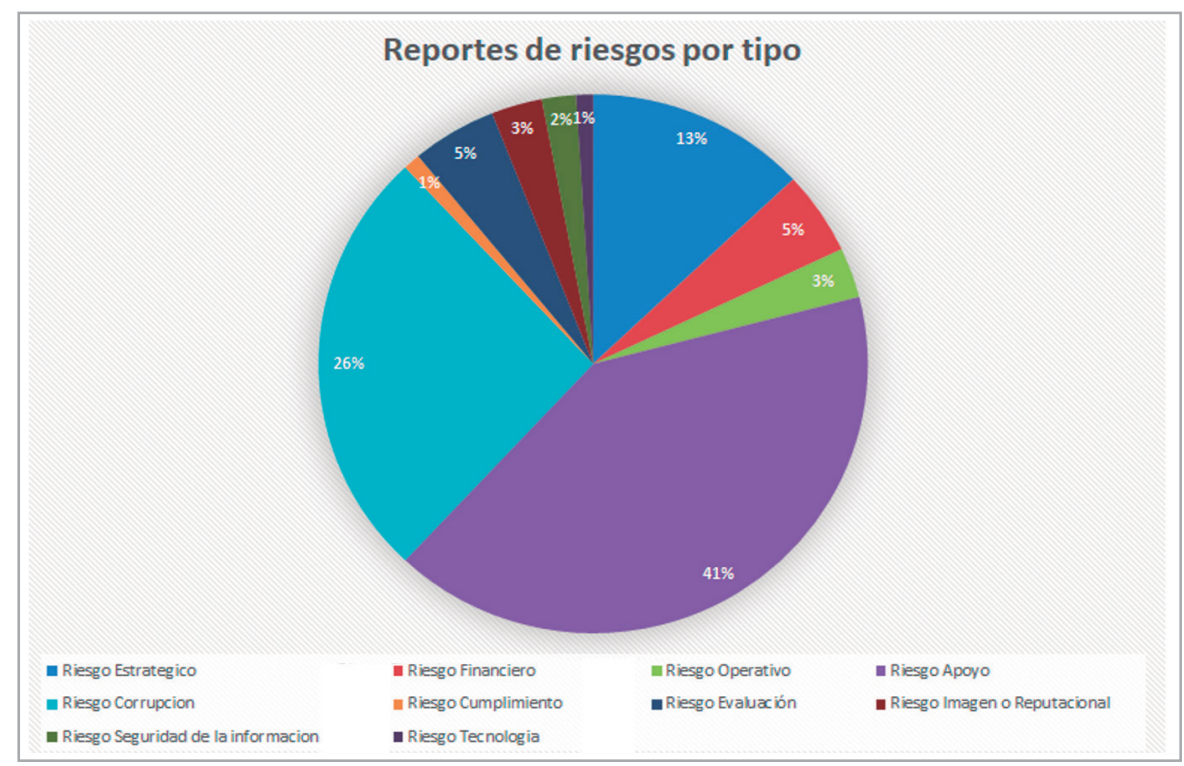

Figura 3. Reportes de riesgos por tipo.

Fuente: Informe anual de riesgos vigencia 2019 (Departamento de Planeación del Ejército Nacional de Colombia, 2020a).

De acuerdo con lo anterior, es importante resaltar el concepto de riesgo residual, que el Departamento Administrativo de la Función Pública (2018) define como el nivel de riesgo que permanece luego de tomar sus correspondientes medidas de tratamiento (p. 8). Esto quiere decir que el riesgo siempre va a existir, sin importar las medidas que se tomen, y que esto solo se podrá evitar gracias a la ética de la persona o de las personas que estén a cargo de estos procesos. Específicamente, el Departamento de Planeación del Ejército Nacional (2020) actualizó — con fecha del 23 de septiembre del 2020_, veintisiete (27) procesos, los cuales cuentan con cincuenta (50) riesgos contemplados (figura 4): 


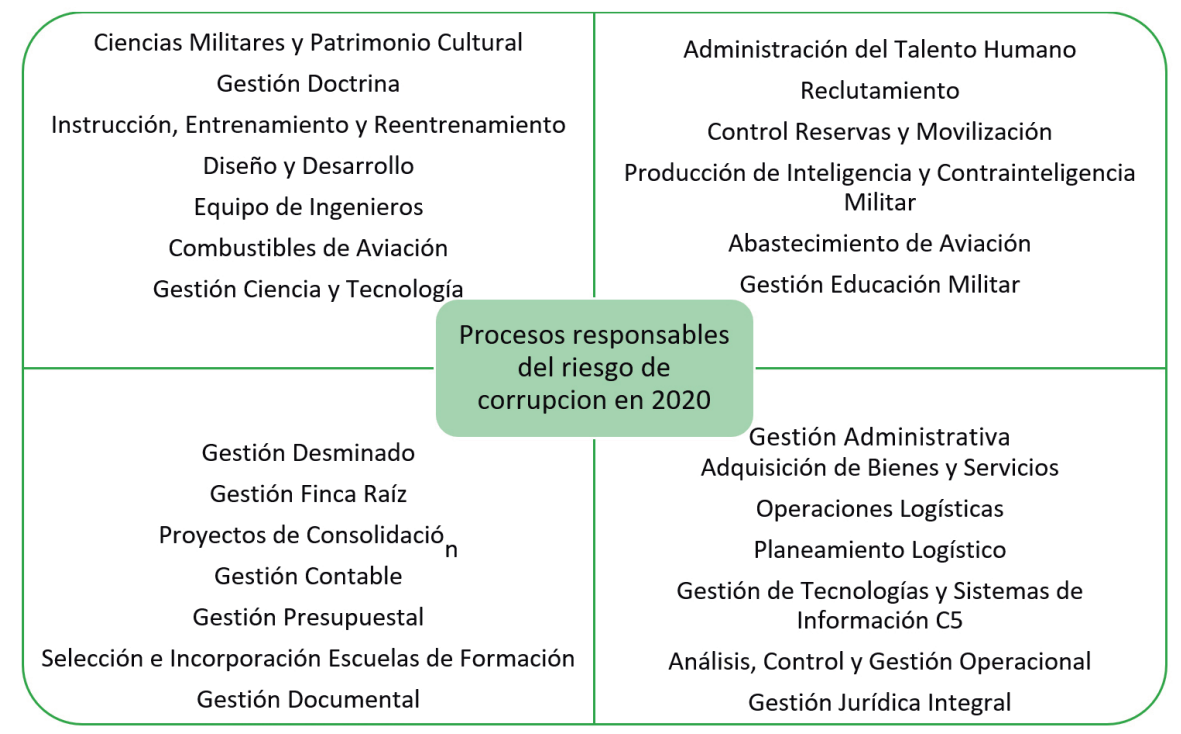

Figura 4. Procesos responsables de riesgo de corrupción en el Ejército Nacional en 2020. Fuente: elaboración propia con base en la Matriz de Contexto Estratégico de Administración de Riesgos del Departamento de Planeación del Ejército Nacional de Colombia (2020b).

$\mathrm{Al}$ situar los riesgos de corrupción de los anteriores procesos en un mapa de calor (figura 5), se obtiene que su impacto en la institución está situado entre moderado y catastrófico. Precisamente, el escenario catastrófico cuenta con una incidencia alta, el mayor con una incidencia media y el moderado con una incidencia baja, lo cual deja ver que sin importar la probabilidad de que ocurra, el impacto para la institución siempre va a ser alto, tanto desde el contexto interno como en el contexto externo.

Aunque el Ejército Nacional cuenta con formas de tratar el riesgo dependiendo del impacto que este genere, en el caso de la corrupción, al ser un riesgo residual, el tratamiento siempre se va a encaminar a reducir las oportunidades de que se materialice. Teniendo en cuenta esto y recordando el numeral 11 del Código de Honor del Soldado Colombiano — "La ética es mi única regla para tomar decisiones”-, el Ejército cuenta con la Dirección de Aplicación de Normas de Transparencia del Ejército (DANTE), la cual tiene la misión de promover la aplicación de normas que generen cambios culturales y pedagógicos enmarcados en la ética y la transparencia como resultado del acompaña- 


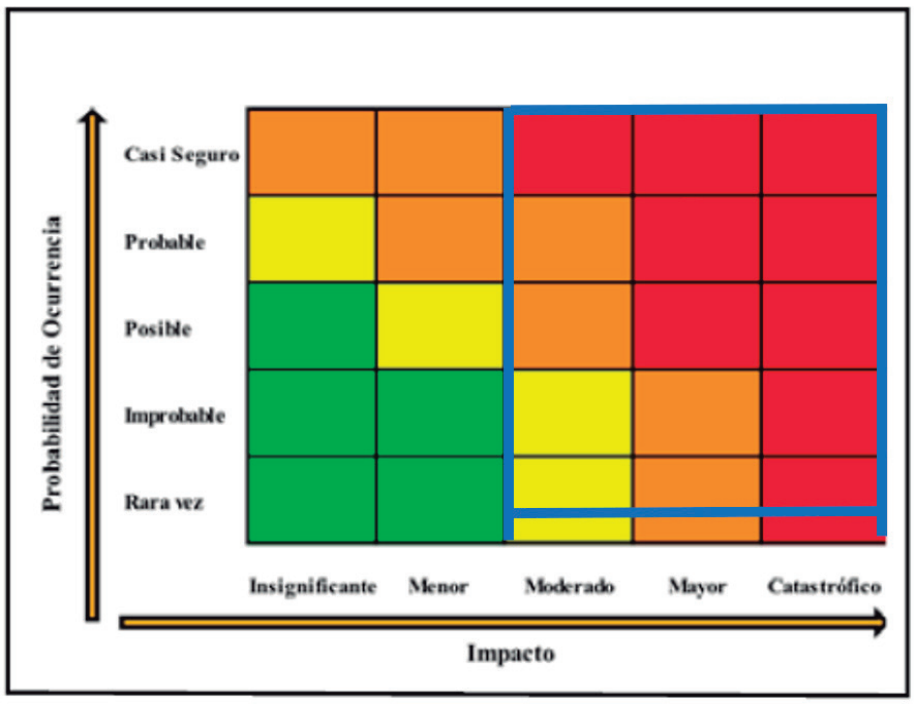

Figura 5. Mapa de calor de los resultados de calificación de corrupción.

Fuente: Informe anual de riesgos vigencia 2019 (Departamento de Planeación del Ejército Nacional de Colombia, 2020b).

miento focalizado preventivo a los diferentes procesos que desarrolla la Fuerza, con el fin de asesorar y recomendar oportunamente al alto mando sobre ajustes y correcciones pertinentes haciendo uso de información privilegiada (Centro de Educación Militar, 2019) y cuenta con las siguientes capacidades:

- Promover la ética y la transparencia institucional.

- Efectuar acompańamiento y monitoreo de nivel estratégico.

- Consolidar y difundir los planes y políticas generados por el comando superior en temas de transparencia y cultura ética.

- Articular el cumplimiento de la gestión con organizaciones afines.

- Mantener comunicación oportuna con el alto mando.

- Promover ajustes y recomendaciones.

- Acceder a información privilegiada (parr. 6).

Esto muestra la importancia que tiene para el Ejército Nacional no solo la administración de riesgos, sino también la capacitación y el fortalecimiento de la ética institucional en los uniformados vinculados a la institución. 


\section{Métodos}

Esta investigación tiene una metodología cualitativa, en el entendido de que este método usa palabras, textos, discursos, dibujos, gráficos e imágenes para construir un conocimiento de la realidad social durante un proceso de construcción y/o comprobación teórica desde una perspectiva amplia, ya que la intención es entender todas las cualidades que se interrelacionan entre sí y que caracterizan un fenómeno determinado. La perspectiva cualitativa de la investigación pretende acercarse a la realidad social a partir de la utilización de datos no cuantitativos (Quecedo \& Castaño, 2002).

Específicamente, la investigación hace uso de una metodología cualitativa que estudia las dimensiones políticas, económicas y sociales del sector defensa y puntualmente dentro de la Fuerza Pública. Además, desde un punto de vista comparativo, se observan las condiciones que hacen más vulnerable a la Fuerza Pública y los factores que afectan la ejecución del presupuesto asignado a la seguridad y defensa, así como las actividades que la complementan.

Asimismo, esta investigación es de carácter inductivo, en el entendido de que el método cualitativo, según De Souza (citado por Naupas, 2014), es adecuado para estudiar las relaciones, las percepciones y las representaciones, que son producto de las interpretaciones que los humanos hacen en relación con cómo viven. Con este propósito, hay que tener presente los espacios formales de la economía y la política (De Souza; citado por Ñaupas, 2014, p. 357) con un alcance descriptivo — como explican Sampieri y Baptista (2014)_, el cual busca especificar las propiedades, las características y los perfiles de personas, grupos, comunidades, procesos, objetos o cualquier otro fenómeno que se someta a un análisis (p. 98).

Teniendo en cuenta esto, la investigación como hecho particular aborda la ética profesional de los uniformados del Ejército Nacional de Colombia en relación con los riesgos y factores susceptibles de corrupción (figura 6). 


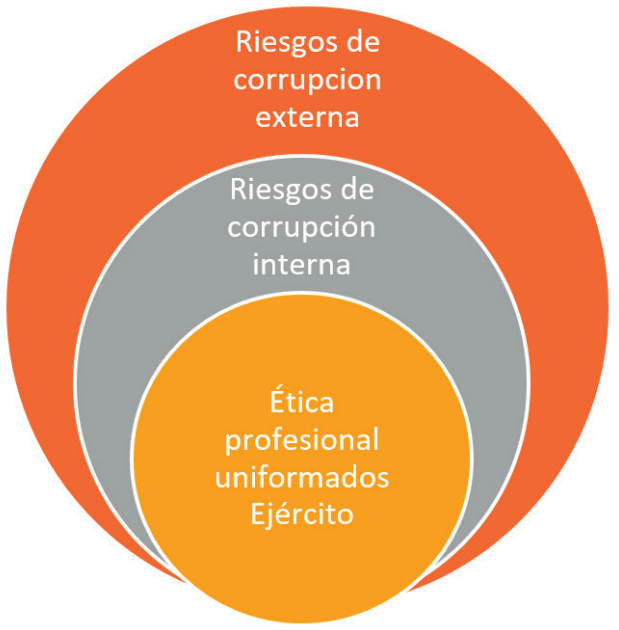

Figura 6. De lo particular a lo general.

Fuente: elaboración propia.

\section{Resultados}

En la primera etapa se realizó una búsqueda de fuentes primarias y secundarias en bases de datos, documentos oficiales y revistas indexadas, para lo cual el criterio de inclusión o exclusión se realizó a través de fichas de Resumen Analítico Especializado (RAE). En la segunda, se realizaron entrevistas semiestructuradas a personal activo y de la reserva activa del Ejército Nacional sobre las susceptibilidades de corrupción y la ética en la Fuerza de acuerdo con categorías apriorísticas (figura 7). Finalmente, la tercera etapa consistió en el análisis de la información recolectada.

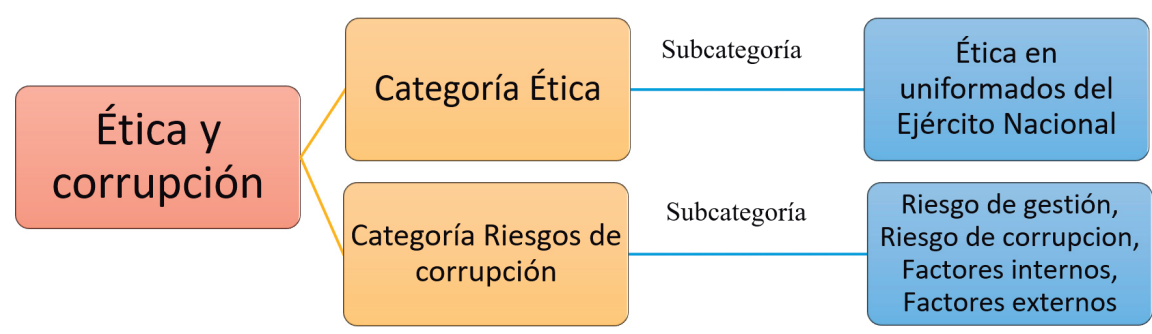

Figura 7. Categorías apriorísticas.

Fuente: elaboración propia. 
Debido a su magnitud, capacidad operativa, cantidad de personas y presupuesto, el Ejército Nacional presenta diferentes riesgos de corrupción. Por esta razón, cuenta con un sistema robusto de administración de riesgos internos, pero hay factores de los contextos externos en los que también existen riesgos de corrupción, entre los cuales el factor social es uno de los más importantes. Específicamente, dentro de los doce factores sociales que se encuentran en la Matriz Estratégica de Riesgos del Departamento de Planeación del Ejército Nacional (2020b) se encuentran cuatro que pueden ayudar a explicar lo que se ha dicho anteriormente:

1. Surgimiento de nuevas amenazas.

2. Fenomenologías generadas por actividades ilegales.

3. Necesidades básicas insatisfechas de la población civil.

4. Desatención en problemáticas de las comunidades lejanas.

Antes de continuar es necesario hacer una precisión. La siguiente explicación no generaliza ni busca estigmatizar a las poblaciones insatisfechas y desatendidas, solo busca explicar cómo las nuevas amenazas y los actores ilegales se aprovechan de algunas personas de estas poblaciones para realizar sus actividades ilícitas.

En varios territorios del país la oferta estatal es suplida por los integrantes de la Fuerza Pública, los cuales, a través de sus programas de prevención, acercamiento a la comunidad y acción integral, han llevado a las comunidades lejanas y con necesidades básicas insatisfechas campañas de salud, de educación, entre otros. También existe presencia de uniformados del Ejército haciendo patrullajes en las diferentes zonas del país o presencia de batallones.

Sin embargo, también existen grupos ilegales que se aprovechan de la vulnerabilidad de la población civil de estas comunidades, en las cuales algunas personas se dejan cautivar de estas y terminan realizando actividades ilícitas en pro de mejorar su calidad de vida.

Entre las alternativas más comunes se encuentra la siembra de cultivos ilícitos, pues según la Oficina de las Naciones Unidas contra la Droga y el Delito (Unodc, 2020), en el año 2019 se reportaron 154.000 hectáreas sembradas con plantas de coca en Colombia. En este contexto, los integrantes 
del Ejército deben apoyar la erradicación de estos cultivos ilícitos, pero durante la erradicación, el patrullaje e incluso en los batallones cercanos a estas zonas se pueden encontrar factores de riesgo externo asociados a las cuatro causas descritas anteriormente y que forman parte del factor social, las cuales fueron explicadas en las entrevistas que se realizaron en esta investigación (figura 8).

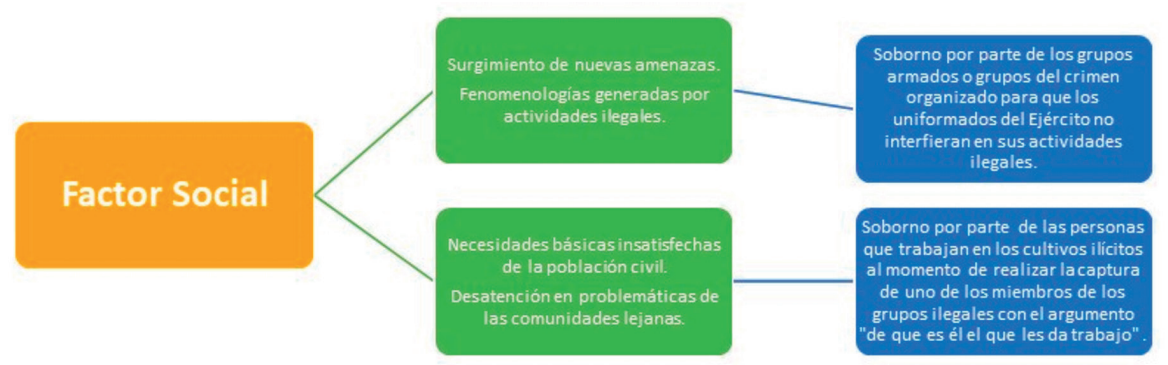

Figura 8. Factores sociales con riesgo de corrupción.

Fuente: elaboración propia con base en las entrevistas realizadas.

$\mathrm{Al}$ igual que los riesgos internos de corrupción, estos factores externos también pueden ser catalogados como un riesgo residual, de manera que la materialización o no depende de la conducta ética del uniformado.

\section{Discusión}

La delgada línea entre el Estado y el sector privado, caracterizada por una creciente externalización de los servicios públicos, puede estar entre las causas estructurales de las prácticas corruptas (Sandoval, 2016). La corrupción estructural sería "una forma específica de dominación social sustentada en un diferencial de poder estructural en el que predominan el abuso, la impunidad y la apropiación indebida de los recursos de la ciudadanía” (p. 123).

Si bien el Ejército Nacional de Colombia tiene claro cuáles son los riesgos de corrupción internos que pueden afectar la institución y ha creado diferentes estrategias para mitigarlos desde el punto de vista preventivo-administrativo, también es importante que realice un sistema de administración de riesgos externos y que tenga en cuenta que si bien estos son cambiantes, hay algunos como los factores sociales en los cuales se pueden hacer análisis en perspectiva para determinar el riesgo para la institución. 
Si bien los procesos administrativos son importantes, también hay que tener en cuenta el factor humano. Por esta razón, el Ejército Nacional ha visto en el fortalecimiento ético uno de los aspectos importantes para reducir los riesgos de corrupción, ya que se trata de un riesgo residual y por tanto va a ser permanente pese a las medidas administrativas que se tomen. En consecuencia, solo queda confiar en que las actividades de fortalecimiento ético realizadas por la institución causen efectos positivos tanto en el personal militar como en la lucha contra la corrupción.

Establecer, instaurar y fortalecer la cultura de la ética desde las escuelas de formación y capacitación es una de las formas de prevención que pueden llegar a ser más efectivas, ya que, después de todo, la intencionalidad de realizar un acto de corrupción se puede materializar sin importar las acciones preventivas de tipo administrativo.

\section{Conclusiones}

No solamente se conceptualiza la corrupción como el resultado de la erosión de valores éticos, sino también como el producto de la pérdida de lazos de solidaridad entre los ciudadanos y el reemplazo por un sistema utilitarista: "El utilitarismo exacerbado, aunado al culto de la viveza, ha generado un fuerte debilitamiento del sentido de lo público y ha conducido a una perspectiva que privatiza el espacio y la función pública” (Salgado, 2004, p. 30). Como consecuencia, la complacencia, la tolerancia y la resignación ante la corrupción predominan sobre amplios sectores de la población (Julián \& Bonavia, 2017).

Los riesgos de corrupción van a ser una problema latente en el Ejército Nacional, si bien se pueden controlar a través de la administración de procesos de riesgo, si algún miembro del Ejército tiene la intencionalidad de cometer una acto de estos, lo podría hacer y más si el riesgo de corrupción es externo; de ahí deviene la importancia de fortalecer la ética en los uniformados del Ejército Nacional para que se regulen y no incurran en hechos de corrupción.

No basta con igualar la ética militar al mismo nivel de la Constitución nacional o mencionarla en el Código de Honor del Soldado Colombiano si esto se queda solo en el papel. El Ejército Nacional no solo debe realizar con sus 
uniformados los ejercicios de memorización correspondientes a la enseñanza de la ética militar, sino que también debe buscar metodologías innovadoras para que estos apropien y sientan ese orgullo de ser un ejemplo para los ciudadanos del país.

\section{Referencias}

Bautista, D. (2005). La ética y la corrupción en la politica y en la administración pública. [Tesis de maestría, Universidad Complutense de Madrid]. Repositorio UCM. https:// eprints.ucm.es/id/eprint/7816/1/tesis-maestr\%C3\%ADa_2.pdf

Boniolo, P. (2010). La trama de corrupción: un estudio en la clase media y la clase trabajadora de Buenos Aires. Revista Mexicana de Sociología, 391-365 ,(3)72.

Centro de Educación Militar [CEMIL]. (2019). Documentos Institucionales. Política DANTE del Ejército Nacional de Colombia. https:/cemil.edu.co/index.php/informacion-deinteres/documentos-institucionales/

Cialdini, R. B., Reno, R. R., \& Kallgren, C. A. (junio, 1990). A focus theory of normative conduct: recycling the concept of norms to reduce littering in public places. Journal of Personality and Social Psychology, 58(6), 1015-1026.

Comando General de las Fuerzas Militares. (2016). Manual de generalidades éticas para la vocación militar. https://www.mindefensa.gov.co/irj/go/km/docs/Mindefensa/ Documentos/descargas/Sobre_el_Ministerio/Control_Interno/documentos/manual_ generalidades_eticas_FFMM.pdf

Departamento de Planeación del Ejército. (2020a). Informe anual de riesgos vigencia 2019. Ejército Nacional de Colombia.

Departamento de Planeación del Ejército. (2020b). Matriz Estratégica de Riesgos. Ejército Nacional de Colombia

Departamento de Planeación del Ejército. (2020c). Riesgos de corrupción 1 y 2. Ejército Nacional de Colombia.

Departamento Nacional de la Función Pública. (2018). Guía para la administración del riesgo y el diseño de controles en entidades públicas. http://www.funcionpublica. gov.co/documents/418548/34150781/Gu\%C3\%ADa+para+la+administraci\%C3 $\% \mathrm{~B} 3 \mathrm{n}+\mathrm{del}+$ riesgo $+\mathrm{y}+\mathrm{el}+\mathrm{dise} \% \mathrm{C} 3 \% \mathrm{~B} 1 \mathrm{o}+\mathrm{de}+$ controles+en+entidades+p $\% \mathrm{C} 3 \% \mathrm{BAbl}$ icas+-+Riesgos+de+gesti $\%$ C3\%B3n $\% 2 \mathrm{C}+$ corrupci $\% \mathrm{C} 3 \% \mathrm{~B} 3 \mathrm{n}+\mathrm{y}+$ seguridad+digital++ Versi\%C3\%B3n+4+-+Octubre+de+2018.pdf/68d324dd-55c5-11e0-9f372e5516b48a87? $t=1542226781163 \&$ download=true

Díaz, Á. (2003). Ética y corrupción. Lo público y la democracia. Convergencia. Revista de Ciencias Sociales, 10(31), 141-151. https://www.redalyc.org/pdf/105/10503107.pdf

Diego Bautista, Ó. (2018). El problema de la corrupción en América Latina y la incorporación de la ética para su solución. Espacios Públicos, 15(35), 48-62. https://www.redalyc.org/ articulo.oa?id=67624803004 
Ejército Nacional de Colombia. (2017). Manual fundamental del Ejército MFE 1.0. https:// www.cedoe.mil.co/centro_doctrina_ejercito_nacional_colombia/doctrina/manuales_ fundamentales_ejercito_mfe/mfe_1_0_ejercito

Ejército Nacional de Colombia. (2020a). Código de ética institucional del Ejército 2020. https:// www.ejercito. mil.co/?idcategoria $=437073 \&$ download $=$ Y.

Ejército Nacional de Colombia. (2020b). Dirección de Aplicación de Normas de Transparencia del Ejército (DANTE). https://www.ejercito.mil.co/conozcanos/organigrama/segundo_ comandante_ejercito_403585/direccion_aplicacion_normas_408436

Estévez, A. (2005). Reflexiones teóricas sobre la corrupción: sus dimensiones política, económica y social. Revista Venezolana de Gerencia, 10(29), 43-85. https://www.redalyc.org/ pdf/290/29002904.pdf

Gino, F., \& Bazerman, M. (2009). Cuando la mala conducta pasa desapercibida: La aceptabilidad de la erosión gradual en el comportamiento poco ético de otros. Revista de Psicología Social Experimental, 45(4), 708-719.

Johnston, M. (2006). Syndromes of Corruption: Wealth, Power, and Democracy. Nueva York: Cambridge University Press.

Julián, M., \& Bonavia, T. (2017). Aproximaciones psicosociales a la corrupción: Una revisión teórica. Revista Colombiana de Psicología, 26(2), 231-243. https://www.redalyc.org/ pdf/804/80454275004.pdf

Mishra, A. (2006). Persistencia de la corrupción: algunas perspectivas teóricas. Desarrollo Mundial, 34(2), 349-358.

Naaupas, H., Mejía, E. M., Ramírez, E. N., \& Paucar, A. V. (2014). Metodología de la investigación cuantitativa-cualitativa y redacción de la tesis. Ediciones de la U.

Oficina de las Naciones Unidas contra la Droga y el Delito [UNODC]. (2020). UNODC's Action against corruption and economic crime. https://www.unodc.org/unodc/en/ corruption/index.html?ref=menuside

Presidencia de la República. (2019). Lucha contra la corrupción, máxima prioridad del Gobierno del Presidente Duque. https://id.presidencia.gov.co/Paginas/prensa/2019/190104-Lucha-contra-la-corrupcion-maxima-prioridad-del-Gobierno-del-Presidente-Duque.aspx

Quecedo, R., \& Castańo, C. (2002). Introducción a la metodología de investigación cualitativa. Revista de Psicodidáctica, (14), 5-39. https://www.redalyc.org/articulo.oa?id=17501402

Salgado Lévano, C. (2004). El flagelo de la corrupción: Conceptualizaciones teóricas y alternativas de solución. Liberabit. Revista Peruana de Psicología, (10), 27-40. https://www. redalyc.org/articulo.oa?id=68601005

Sampieri, R., Fernández Collado, C., \& Baptista Lucio, M. D. (2014). ¿Qué características posee el enfoque cuantitativo de investigación? En Metodología de la investigación (p. 4). McGraw-Hill/Interamericana.

Sandoval , I. E. (2016). Enfoque de la corrupción estructural: Poder, impunidad y voz ciudadana. Revista Mexicana de Sociología, (78), 119-152. 
Transparencia por Colombia. (2019a). Brief de la corrupción en Colombia. https:// transparenciacolombia.org.co/18/01/2019/brief-de-la-corrupcion-en-colombia/

Transparencia por Colombia. (2019b). ¿Qué es la corrupción? https://transparenciacolombia. org.co/27/08/2019/corrupcion/

Vicuña, L., Hernández, H., Paredes, M., Rivera, J., Ríos, J., Santillana, C., \& Torres, J. (2006). Percepción, tipos y medidas de control de la corrupción según el sexo, ciclo académico y la facultad a la que pertenecen los estudiantes universitarios. Revista Iipsi, 91-65, (2)9. 